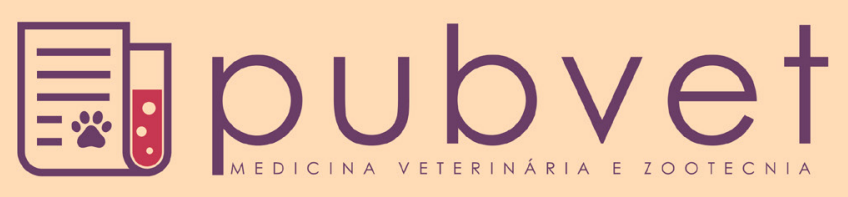

HTTP://DX.DOI.ORG/10.22256/PUBVET.V11N7.652-657

\title{
Qualidade da água proveniente de poço artesiano em viveiro de piscicultura
}

\author{
Fabricio Nilo Lima da Silva ${ }^{1 *}$, Luciano Ramos de Medeiros ${ }^{2}$, Maria Sintia Monteiro da \\ Costa $^{3}$, Antonia Rafaela Gonçalves Macedo ${ }^{4}$, Lian Valente Brandão ${ }^{5}$, Raimundo Aderson \\ Lobão de Souza ${ }^{6}$
}

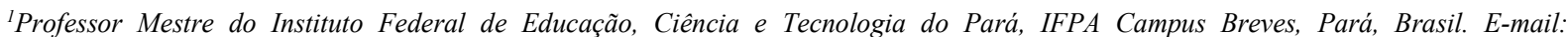
fabricionnilo@hotmail.com;

${ }^{2}$ Mestre em Desenvolvimento Rural e Gestão de Empreendimentos Agroalimentares pelo Instituto Federal de Educação, Ciência e Tecnologia do Pará, IFPACampus Castanhal, Pará, Brasil.E-mail: lucianomedeiros_ses@hotmail.com;

${ }^{3}$ Mestra em Aquicultura e Recursos Aquáticos Tropicais pela Universidade Federal Rural da Amazônia, UFRA Campus Belém, Pará, Brasil. E-mail: sintiamonteiro@hotmail.com:

${ }^{5}$ Doutoranda do Programa de Pós-Graduação em Ciência Animal pela Universidade Federal do Pará, UFPA Campus Castanhal, Pará, Brasil.E-mail: agmaquicultura@hotmail.com;

${ }^{5}$ Professor Doutor do Instituto Federal de Educação, Ciência e Tecnologia do Pará, IFPA Campus Castanhal, Pará, Brasil. E-mail: lianpesca@yahoo.com.br:

${ }^{6}$ Professor Doutor da Universidade Federal Rural da Amazônia, UFRA Campus Belém, Pará, Brasil. E-mail: adersonlobao@globo.com;

*Autor para correspondência
\end{abstract}

RESUMO. O objetivo deste trabalho foi avaliar a qualidade da água em um viveiro de criação semi-intensiva em policultivo das espécies tilápia (Oreochromis niloticus), curimatã (Prochilodus lineatus) e surubim (Pseudoplatystoma sp.) abastecido com água de poço. As análises foram aferidas dentro de um viveiro escavado apresentando tamanho de $3200 \mathrm{~m}^{2}$ de lâmina d'água e 1,5 metros de profundidade, com declividade de 5\%. Durante os 60 dias de cultivo, a cada cinco dias, em dois pontos de coletas, pela manhã (08h00) e à tarde (17h00), aferindo-se os níveis de oxigênio dissolvido na água, temperatura, transparência, potencial hidrogeniônico e amônia total. Para verificar as diferenças estatísticas significativas entre os pontos de coleta sobre a qualidade de água, as médias foram submetidas à análise de variância (ANOVA) ao nível de 5\%. Durante o experimento, as variáveis mantiveram-se dentro dos limites estabelecidos como satisfatórios para o cultivo de peixes tropicais de água doce, não apresentando diferenças entre os pontos de coletas.

Palavras chave: Piscicultura, Policultivo, Amazônia

\section{Quality of water from artesian wells in fish farms}

ABSTRACT. The objective of this study was to evaluate the quality of water in a semiintensive breeding tank in policultive of the species tilapia (Oreochromis niloticus), curimata (Prochilodus lineatus) and surubim (Pseudoplatystoma sp.) stocked with well water. The analyses were gauged inside a hollowed tank presenting size of $3200 \mathrm{~m}^{2}$ water layer and 1,5 meters of depth, with steepness of $5 \%$. During the 60 days of culture, every five days, in two collection points about the water quality, the averages were subjected to analysis of variance (ANOVA) at 5\% level. During the experiment, the variables were maintained within the established limits were satisfactory for the fish cultive of sweet water, did not show differences between the gathering points.

Keywords: Island, community, agriculture, alternative, fish farming 


\section{Calidad del agua proveniente de pozo artesanal en vivero de piscicultura}

RESUMO. El objetivo de este trabajo fue evaluar la calidad del agua en un sistema de cría semi intensivo en policultivo de Oreochromis niloticus, Prochilodus lineatus y Pseudoplatystoma sp. abastecido con agua de pozo artesanal. Los análisis fueron realizados dentro de un vivero escavado que presentaba tamaño de $3200 \mathrm{~m}^{2}$ de lámina de agua e 1,5 metros de profundidad, con declividad de 5\%. Durante los 60 días de cultivo, a cada cinco días, en dos puntos de colectas, por la mañana $(08 \mathrm{~h} 00)$ y en la tarde $(17 \mathrm{~h} 00)$, midiéndose los niveles de oxígeno disuelto en el agua, temperatura, transparencia, potencial hidrogeno y amoníaco total. Para verificar las diferencias estadísticas entre los puntos de colecta sobre la calidad del agua, los promedios fueron sometidos a análisis de varianza (ANOVA) a nivel de 5\%. Durante el experimento, las variables se mantuvieron dentro de los límites establecidos como satisfactorios para el cultivo de peces tropicales de agua dulce, no presentando diferencias entre los puntos de colecta.

Palabras clave: Piscicultura, policultivo, amazonia

\section{Introdução}

O policultivo na aquicultura é um sistema integrado de criação, no qual duas ou mais espécies aquáticas são criadas no mesmo local (Costa et al., 2013). Na piscicultura continental, a qualidade da água vem tomando impulso de grande interesse neste setor produtivo, uma vez que, em um determinado ambiente, a água em condições inadequadas acarretará problemas no cultivo, levando os peixes à morte (Lachi and Sipaúba-Tavares, 2008).

A disponibilidade e qualidade da água são fatores fundamentais, por conseguinte, diversos são os casos de perdas econômicas relacionadas ao descuido de produtores com relação à qualidade de água (Mallasen et al., 2008). Em viveiros escavados, existem poucas informações a respeito da utilização de água de poço em policultivos. Por conta disso, há necessidade de estudos limnológicos que contribuam para a melhor caracterização da água nessas atividades aquícolas, avaliando a natureza de alguns problemas e avaliando as possíveis ações de manejo que possa gerar um meio ambiente mais sustentável (Santeiro, 2005).

Por este motivo, torna-se necessário o planejamento para manter o controle da qualidade de água nos viveiros onde organismos aquáticos estão sendo cultivados; atingindo desta forma, um bom desenvolvimento e consequentemente, uma produção economicamente viável (Stachiw et al., 2014). De acordo com Sipaúba-Tavares (1995), as variáveis físico-químicas mais apropriadas à qualificação da água de viveiros são: oxigênio dissolvido; $\mathrm{pH}$; dióxido de carbono livre; alcalinidade total; dureza; condutividade elétrica; temperatura; transparência; nutrientes e abundância de plâncton.

Neste contexto, o policultivo se encaixa nos princípios da aquicultura sustentável, uma vez que visa reduzir o impacto ambiental da atividade, melhorando a eficiência de alimentação, a qualidade da água e aumentando a renda dos produtores (Martínez-Porchas et al., 2010). O conhecimento da qualidade da água é de fundamental importância para a aquicultura, sendo imprescindível em trabalhos de criação de peixes (Castro et al., 2014), principalmente em sistema de policultivo. Assim, água na criação dos peixes neste sistema é um aspecto fundamental para garantir seu bom desempenho zootécnico e sobrevivência.

O objetivo deste trabalho foi avaliar a qualidade da água em um viveiro de criação semiintensiva em policultivo das espécies tilápia, Oreochromis niloticus, curimatã Prochilodus lineatus e surubim, Pseudoplatystoma sp. abastecido com água de poço artesiano.

\section{Material e Métodos}

O presente trabalho foi realizado na Unidade de Produção e Pesquisa em Piscicultura de Água Doce (Setor de Piscicultura) do Instituto Federal de Educação, Ciência e Tecnologia do Pará (IFPA) Campus Castanhal ( $1^{\circ} 17^{\prime} 46^{\prime \prime} \mathrm{S}$ e $47^{\circ} 55^{\prime}$ 28" O), no período de dezembro de 2014 a fevereiro de 2015, ao longo de 60 dias de cultivo.

As análises dos parâmetros físicos e químicos da água foram aferidas dentro de um viveiro 
escavado apresentando tamanho de $3.200 \mathrm{~m}^{2}$ de lâmina d'água e 1,5 metros de profundidade, com declividade de $5 \%$, abastecido por água de poço artesiano. $\mathrm{O}$ viveiro em estudo apresenta sistema de criação semi-intensiva em policultivo das espécies tilápia, Oreochromis niloticus, curimatã Prochilodus lineatus e surubim, Pseudoplatystoma sp.

Foram aferidos os parâmetros de qualidade da água, sendo realizadas a cada (cinco dias), em dois pontos de coletas (A - obtidas próximo à fonte de água que abastece o viveiro, $\mathrm{B}$ - próximo à saída de água). As amostras de água foram colhidas com garrafa de Van Dorn (5 L) para determinar os níveis de amônia total mediante um kit técnico laboratorial segundo Lopes et al. (2010), tanto pela manhã ( $08 \mathrm{~h} 00)$ e à tarde (17h00), aferindo-se também os níveis de oxigênio dissolvido na água $(\mathrm{mg} / \mathrm{L})$ (média \pm desvio) e temperatura $\left({ }^{\circ} \mathrm{C}\right)$ (média \pm desvio) pelo aparelho eletrônico Oxímetro/Termômetro (ICEL manaus $^{\circledR}$ modelo $O D-4000$ ), a transparência (média \pm desvio) foi avaliada pelo disco de Secchi, o potencial hidrogeniônico (média \pm desvio) e a condutividade (média \pm desvio) foram aferidas por uma caneta multiparâmetro digital.

Os resultados foram comparados com os padrões das Resoluções do CONAMA $n^{\circ}$ $\underline{357 / 2005}$ e $\mathrm{n}^{\circ} 430 / 2011$, para verificar se a qualidade da água era compatível com o uso para sistemas de criação. Para verificar as diferenças estatísticas significativas entre os pontos de coleta sobre a qualidade de água, as médias foram submetidas à análise de variância ao nível de 5\%.

\section{Resultados e Discussão}

A figura 1 apresenta as variáveis da qualidade da água no viveiro mantendo dentro dos limites estabelecidos como satisfatórios para o cultivo de peixes tropicais de água doce, não apresentando diferenças $(P>0,05)$ entre os pontos de coleta.

A pesquisa revelou que o teor de oxigênio dissolvido na água foi, em média $3,15 \pm 0,44 \mathrm{mg} / \mathrm{L}^{-}$ ${ }^{1}$ e $5,84 \pm 0,26 \mathrm{mg} / \mathrm{L}^{-1}$ no período da manhã e da tarde, respectivamente. De acordo com Chang and Ouyang (1988), em dias claros a taxa fotossintética aumenta, elevando a demanda de oxigênio a noite e acarretando num déficit desse gás pela manhã. Assim, os valores do presente estudo estão acima do nível mínimo de oxigênio dissolvido para tambaquis $\left(3,0 \mathrm{mg} / \mathrm{L}^{-1}\right)$, citado por Souza and Teixeira Filho (1985).
A temperatura da água, está se destaca como um fator importante no cultivo de peixes por influenciar diretamente na ingestão de alimentos (Frasca-Scorvo et al., 2007), onde o consumo é reduzido ou mesmo cessado com a variação da temperatura da água para além da sua faixa ideal (Schmittou and Akiyama, 1993). Apesar da elevação da temperatura nos tanques, os valores permaneceram dentro do estabelecido pela Resolução do CONAMA n ${ }^{\circ} 430 / 2011\left(<40^{\circ} \mathrm{C}\right)$. A temperatura média da água foi de $27,86 \pm 0,67^{\circ} \mathrm{C}$ no período da manhã e $28,60 \pm 1,48{ }^{\circ} \mathrm{C}$ no período da tarde, situando-se dentro da faixa ótima (25 a $29^{\circ} \mathrm{C}$ ) estabelecida também por Souza and Teixeira Filho (1985), Kubitza (2009), Sandoval Jr et al. (2013).

Todos os valores de $\mathrm{pH}$ das amostras estavam de acordo Resolução do CONAMA n ${ }^{\circ}$ 430/2011. $\mathrm{Na}$ literatura de Castagnolli (1992), Boyd (2000) e Kubitza (2009), a faixa de $\mathrm{pH}$ próxima de 5,0 a 8,0 é considerada ideal para peixes tropicais. Neste sentido, na presente pesquisa, os valores mantiveram-se entre $7,63 \pm 0,75$ no período da manhã e $8,93 \pm 0,49$ no período da tarde. De acordo com Ceccarelli et al. (2000), grandes concentrações de vegetais, algas e fitoplâncton provocam acidificação do meio aquático à noite, ao passo que altas temperaturas podem acelerar o processo de fotossíntese elevando consideravelmente os valores de $\mathrm{pH}$ à tarde, que posteriormente se tornarão críticos durante a madrugada.

Com relação à condutividade elétrica as médias foram de $35,26 \pm 1,67 \mu \mathrm{S} . \mathrm{cm}^{2}$ no período da manhã e $35,45 \pm 0,59 \mu \mathrm{S} . \mathrm{cm}^{2}$ no período da tarde, apontando o aumento da concentração de íons presente na água do viveiro, porém, os valores máximos permaneceram abaixo de $100 \mu \mathrm{S} / \mathrm{cm}$, o que é muito importante, uma vez que níveis superiores a este indicam ambientes impactados (Cetesb, 1988).

A concentração da forma tóxica da amônia aumenta com a elevação do $\mathrm{pH}$ e da temperatura, de modo que, acima de $0,01 \mathrm{mg} / 1$ passa a afetar o crescimento e a resistência a doenças (Imbiriba et al., 2000). A amônia total, provavelmente, não interferiu no resultado do experimento, também permaneceu próxima aos valores estabelecidos para águas destinadas a produção de organismos aquáticos, que é de $1,0 \mathrm{mg} / \mathrm{L}^{-1}$ (Mardini and Mardini, 2000, Kubitza, 2009). A amônia é o principal resíduo nitrogenado excretado pelos peixes, resultante do metabolismo proteico, e 
contribui para o aumento da decomposição microbiana de resíduos orgânicos (restos de alimentos, fezes e adubos orgânicos).

\section{Oxigênio Dissolvido}

$\mathrm{mg} / \mathrm{L}$

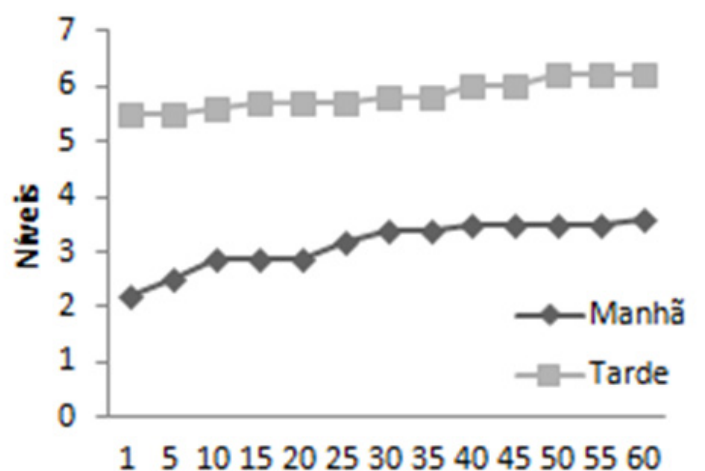

Perido/Dias de análise

pH

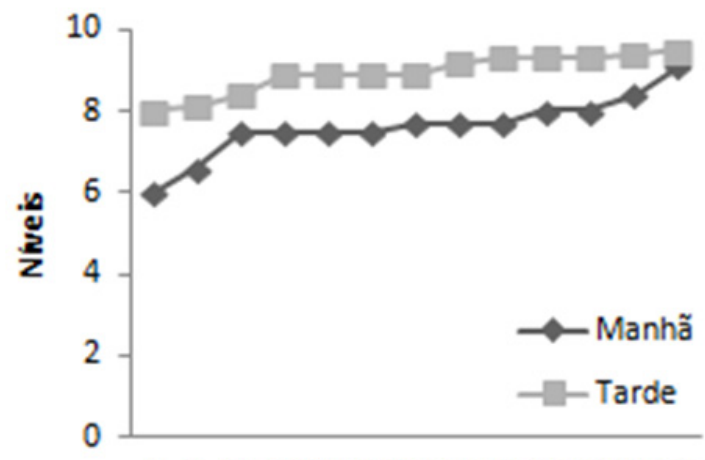

151015202530354045505560

Perído/Dias de análise

Amônia total

$\mathrm{mg} / \mathrm{L}$

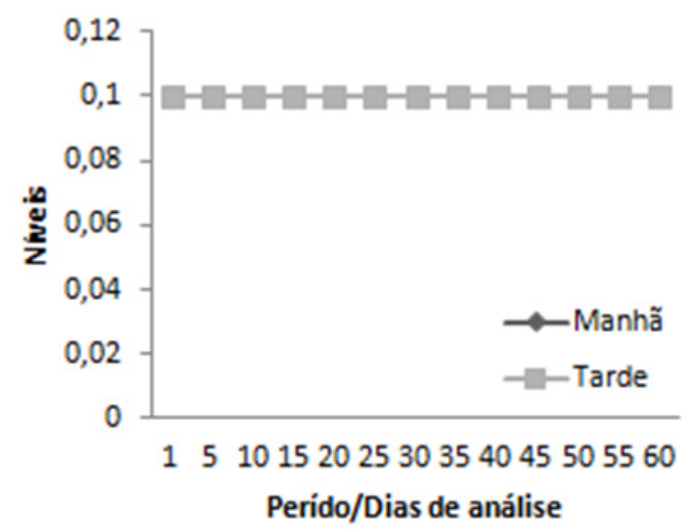

Temperatura

${ }^{\circ} \mathrm{C}$

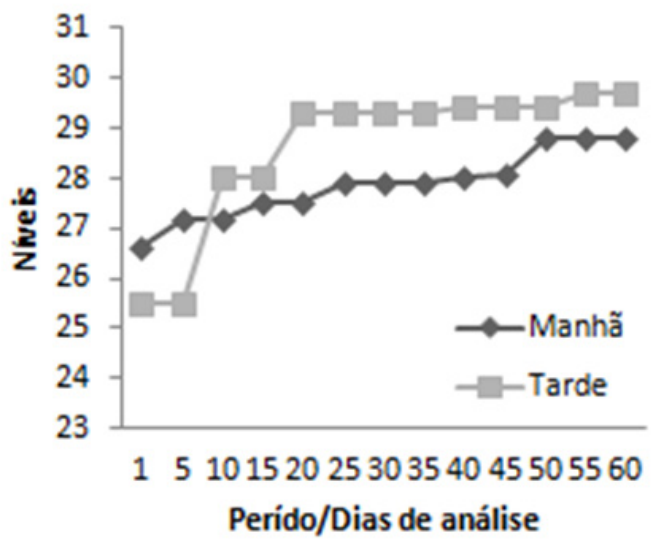

Condutividade

$\mu \mathrm{S} . \mathrm{cm}^{2}$

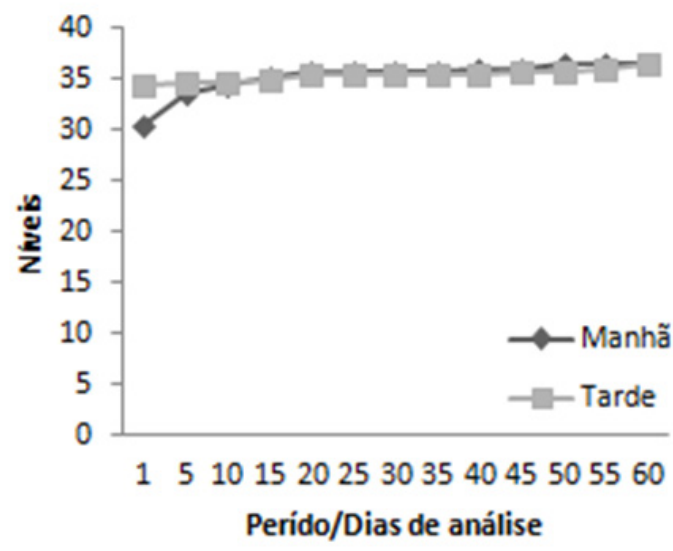

Transparência

$\mathrm{Cm}$

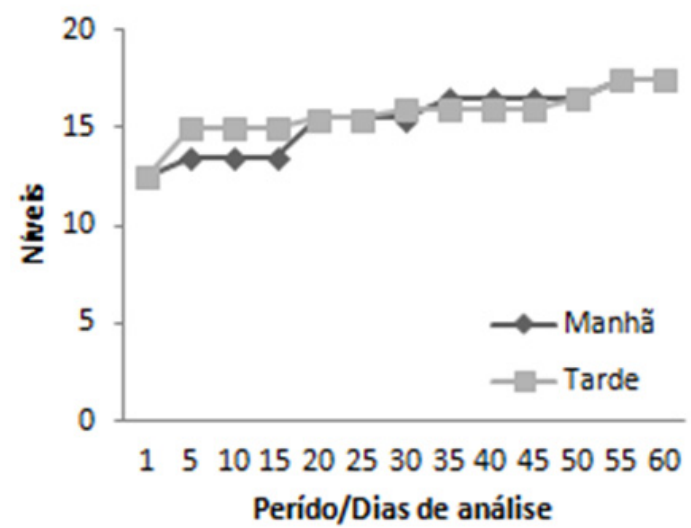

Figura 1: Avaliação da qualidade de água durante 60 dias de cultivo semi-intensiva (policultivo) das espécies tilápia (Oreochromis niloticus), curimatã (Prochilodus lineatus) e surubim (Pseudoplatystoma sp.).

Ceccarelli et al. (2000), a visibilidade do disco de Secchi entre 0,30 e $0,60 \mathrm{~m}$ é ideal para o cultivo de peixes. A transparência da água, durante todo período analisado no presente trabalho, apresentou 
valores de $(12,5 \pm 0,71$ a $17,5 \pm 0,71 \mathrm{~cm})$, estando inferior dos valores recomendados para viveiros de piscicultura 30 a $50 \mathrm{~cm}$ (Kubitza, 2009). Segundo este mesmo autor, águas com transparência elevada possibilitam a maior penetração da luz, favorecendo o crescimento de plantas aquáticas e algas filamentosas, que contribuem para diminuição do oxigênio dissolvido.

\section{Conclusão}

Durante o período de 60 dias de cultivo em sistema semi-intensivo com policultivo das espécies tilápia, curimatã e surubim, abastecido com água de poço artesiano, as variáveis da qualidade da água no viveiro mantiveram-se dentro dos limites estabelecidos como satisfatórios para o cultivo de peixes tropicais de água doce, não apresentando diferenças entre os pontos de coletas.

\section{Referências bibliográficas}

Brasil, (2005) Conselho Nacional do Meio Ambiente. Resolução $n^{\circ}$ 257/2005, de 17 de março de 2005. Dispõe sobre a classificação dos corpos de água e diretrizes ambientais para o seu enquadramento, bem como estabelece as condições e padrões de lançamento de efluentes, e dá outras providências. Publicada no DOU $n^{\circ}$ 053, de 18 de março, p. 58-63.

. Conselho Nacional do Meio Ambiente. Resolução $\mathrm{n}^{\circ} 430$ de 13 de maio de 2011. Dispõe sobre as condições e padrões de lançamentos de efluentes complementam e altera a Resolução CONAMA $\mathrm{n}^{\circ}$ 357/05. Brasília-DF.

Boyd, C. E. 2000. Water quality: an introduction. Springer Science \& Business Media.

Castagnolli, N. 1992. Piscicultura de água doce. FUNEP, Jaboticabal, São Paulo.

Castro, F. S., Nascimento Lobato, A. \& Barbosa, N. D. 2014. Estrutura física e qualidade da água em pisciculturas no município de Macapá-AP-Brasil. Revista de Ciências da Amazônia, 2, 1-10.

Ceccarelli, P. S., Senhorini, J. A. \& Volpato, G. 2000. Dicas em piscicultura; perguntas $e$ respostas. Santana Gráfica Editora, Botucatu.

Cetesb - Companhia de Tecnologia de Saneamento Ambiental. (1988) Parâmetros Físico-Químicos: Importância Sanitária e Parâmetros de Controle. São Paulo.
Chang, W. Y. B. \& Ouyang, H. 1988. Dynamics of dissolved oxygen and vertical circulation in fish ponds. Aquaculture, 74, 263-276.

Costa, L. C. O., Xavier, J. A. A., Neves, L. F. M., Azambuja, A. M. V., Wasielesky Junior, W. \& Figueiredo, M. R. C. 2013. Polyculture of Litopenaeus vannamei shrimp and Mugil platanus mullet in earthen ponds. Revista Brasileira de Zootecnia, 42, 605-611.

Frasca-Scorvo, C. M., Carneiro, D. J. \& Malheiros, E. B. 2007. Efeito do manejo alimentar no desempenho do matrinxã Brycon amazonicus em tanques de cultivo. Acta Amazônica, 621-628.

Imbiriba, E. P., Lourenço Júnior, J. B. \& Moura Carvalho, L. O. D. 2000. Parâmetros ambientais e qualidade de água na piscicultura. EMBRAPA, 8, 1-4.

Kubitza, F. 2009. Manejo na produção de peixes. Panorama da Aqüicultura, 19, 14-23.

Lachi, G. B. \& Sipaúba-Tavares, L. H. 2008. Qualidade da água e composição fitoplanctônica de um viveiro de piscicultura utilizado para fins de pesca esportiva e irrigação. Boletim do Instituto de Pesca, 34, 29-38.

Lopes, J. M., Pascoal, L. A. F., Silva Filho, F. P., Santos, I. B., Watanabe, P. H., Araujo, D. d. M., Pinto, D. C. \& Oliveira, P. S. 2010. Farelo de babaçu em dietas para tambaqui. Revista Brasileira de Saúde e Produção Animal, 11.

Mallasen, M., Barros, H. P. \& Yamashita, E. Y. 2008. Produção de peixes em tanques-rede e a qualidade da água. Revista Tecnologia \& Inovação Agropecuária, 1, 47-51.

Mardini, C. V. \& Mardini, L. B. 2000. Cultivo de peixes e seus segredos. Ulbra, Canoas, Rio Grande do Sul.

Martínez-Porchas, M., Martínez-Córdova, L. R., Porchas-Cornejo, M. A. \& López-Elías, J. A. 2010. Shrimp polyculture: a potentially profitable, sustainable, but uncommon aquacultural practice. Reviews in Aquaculture, 2, 73-85.

Sandoval Jr, P., Trombeta, T. D. \& Mattos, B. O. 2013. Manual de criação de peixes em taquesrede. EMBRAPA, Brasília.

Santeiro, R. M. 2005. Impacto ambiental da piscicultura na qualidade da água e na comunidade planctônica. Centro de Aqüicultura. Universidade Estadual Paulista "Júlio de Mesquita Filho", Jaboticabal. 
Schmittou, H. R. \& Akiyama, D. M. 1993. High density fish culture in low volume cages. American Soybean Association.

Sipaúba-Tavares, L. H. 1995. Limnologia aplicada à aquicultura. Funep, Jaboticabal, São Paulo.

Souza, E. C. P. M. \& Teixeira Filho, A. R. 1985. Piscicultura fundamental. Nobel, São Paulo.

Stachiw, R., Silva, F. R., Vendrusculo, J., Medeiros, T. F., Tavares, V. C. C. \& Dutra, A. R. 2014. Qualidade da água de tanques de piscicultura em Rolim de Moura-RO. Revista Brasileira de Ciências da Amazônia, 2, 22-34.

Article History:

Received 10 February 2017

Accepted 4 May 2017

Available on line 13 May 2017

License information: This is an open-access article distributed under the terms of the Creative Commons Attribution License 4.0, which permits unrestricted use, distribution, and reproduction in any medium, provided the original work is properly cited. 